\title{
Flare of Antiphospholipid Syndrome in the Course of COVID-19
}

\author{
Alexandre Thibault Jacques Maria ${ }^{1,2}$ Isabelle Diaz-Cau ${ }^{3}$ Jean-Marc Benejean ${ }^{4}$ Anaïs Nutz ${ }^{5}$ \\ Aurélie Schiffmann ${ }^{1}$ Christine Biron-Andreani ${ }^{3}$ Philippe Guilpain ${ }^{1,2}$
}

\footnotetext{
1 Department of Internal Medicine-Multi-organ Diseases, Local Referral Center for Auto-immune Diseases, Montpellier School of Medicine, Saint-Eloi University Hospital, University of Montpellier, Montpellier, France

2 IRMB, Inserm U1183, CHU Montpellier (Saint-Eloi University Hospital), University of Montpellier, Montpellier, France

${ }^{3}$ Hemostasis Laboratory, Referral Center for Hemophilia, Montpellier University Hospital, Saint-Eloi University Hospital, Montpellier, France

${ }^{4}$ Department of Radiology, Narbonne Hospital, Narbonne, France

${ }^{5}$ Department of Medicine and Endocrinology, Narbonne Hospital, Narbonne, France
}

TH Open 2020;4:e207-e210.

\author{
Address for correspondence Alexandre Maria, MD, PhD, Department \\ of Internal MedicineMulti-organ Diseases, Saint-Eloi University \\ Hospital, Montpellier F-34295, France \\ (e-mail: a-maria@chu-montpellier.fr).
}

\begin{abstract}
Keywords

- COVID-19

- infection

- thrombosis

- antiphospholipid syndrome

- hydroxychloroquine

We report the case of a 48-year-old man followed since 2013 for primary antiphospholipid syndrome (APLS) revealed by venous thromboembolism in the presence of antiphospholipid antibodies (APL-Abs, anticardiolipin and anti- $\beta$-2-glycoprotein-1), who decompensated in the course of coronavirus disease (COVID-19). Despite efficient anticoagulation, he suffered bilateral adrenal glands hemorrhage and limb arterial ischemia. The tropism of severe acute respiratory syndrome coronavirus- 2 for endothelium can lead to microangiopathy and increased risk for thrombosis. If APL-Abs positivity can be an epiphenomenon under inflammatory and prothrombotic conditions, COVID-19 was herein responsible for disseminated thrombosis and a threat of catastrophic APLS, despite efficient anticoagulation.
\end{abstract}

Antiphospholipid syndrome (APLS) is an autoimmune systemic disorder characterized by thrombosis (involving arteries, veins, and/or small vessels) and/or obstetrical events (such as recurrent early pregnancy loss, fetal loss, or pregnancy morbidity) in association with persistent antiphospholipid antibodies (APL-Abs). ${ }^{1}$ The new emerging coronavirus called "severe acute respiratory syndrome coronavirus-2" (SARS-CoV-2), responsible for the related disease COVID-19 has been reported as a de novo coagulopathy in the setting of APL-Abs. ${ }^{2}$ Herein, we report a flare of APLS following COVID-19, suggesting that SARS-CoV-2 may also trigger thrombosis in pre-existing conditions.

A 48-year-old man treated since 2013 with vitamin $\mathrm{K}$ antagonists (VKA) for primary APLS revealed by venous thromboembolic event (VTE) in the presence of lupus anti- coagulant (LA) and persistent APL-Abs (anticardiolipin [aCL] and anti- $\beta 2$-glycoprotein-1 [aß2GPI], immunoglobulin [Ig]M and $\operatorname{IgG}>40 \mathrm{UI} / \mathrm{L}$ ). The medical history included nongenetic iron overload managed by venesection therapy between 2011 and 2018.

On March 20, 2020, he declared fever, cough, and myalgia, leading to hospitalization on March 25 (day 5). Nasopharyngeal swab was positive for SARS-CoV-2 and computed tomography scan showed typical diffuse lung involvement, without respi-

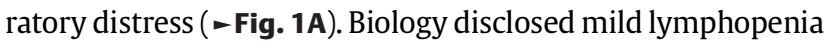
$(0.75 \mathrm{G} / \mathrm{L})$, an elevation of C-reactive protein $(112 \mathrm{mg} / \mathrm{L})$, the platelet count was normal (171 G/L), and INR 2.47 under fluindione. Arterial blood gas tests on ambient air showed mild hypoxemia $\left(\mathrm{pO}_{2} 75 \mathrm{~mm} \mathrm{Hg}\right)$, and normal oxygen saturation (96\%). received

May 3, 2020

accepted after revision

August 12, 2020
DOI https://doi.org/

10.1055/s-0040-1716735. ISSN 2512-9465. (c) 2020 Georg Thieme Verlag KG
Stuttgart · New York

License terms

(c) (i) 

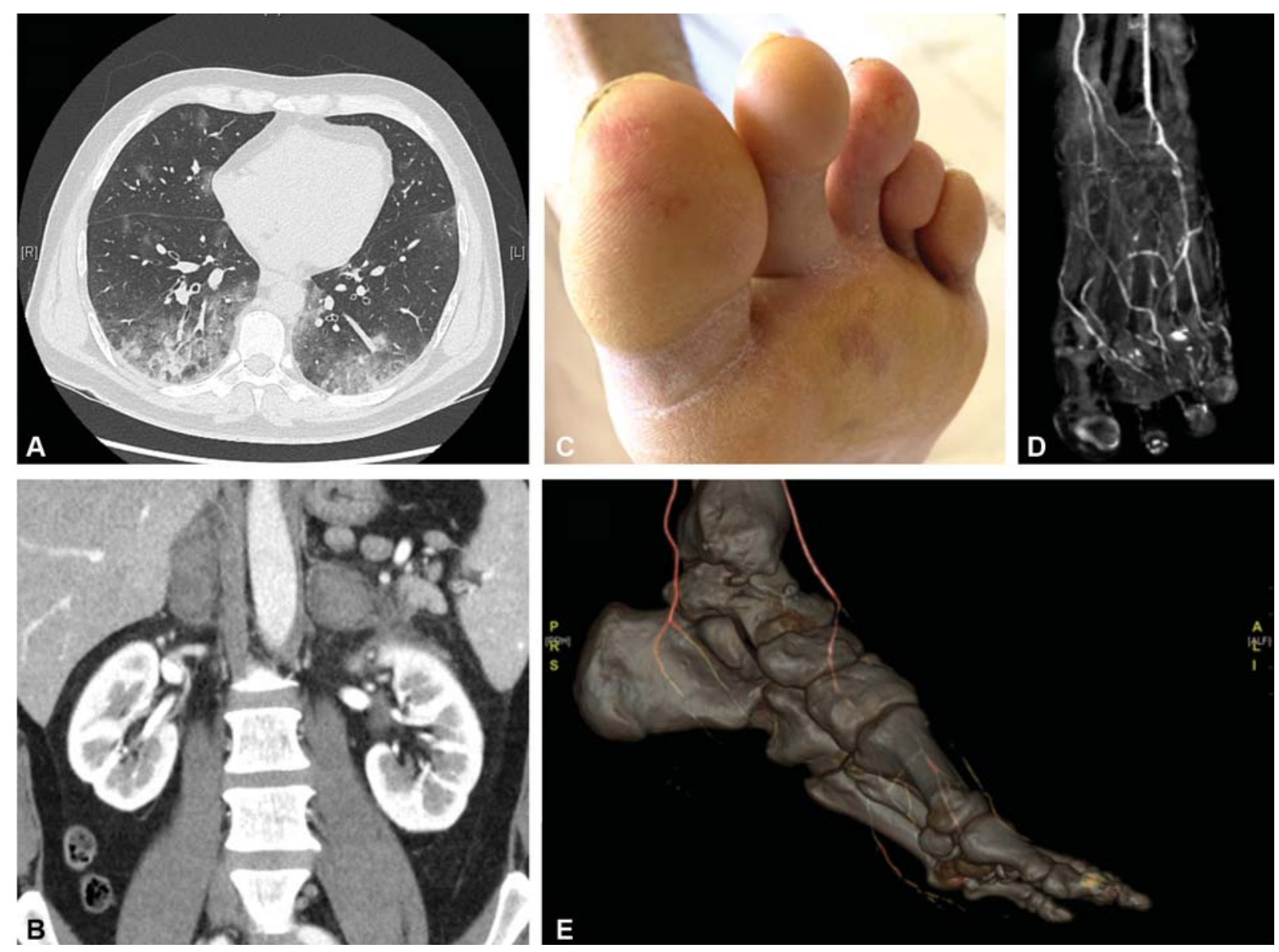

Fig. 1 (A) CT scan disclosing ground glass opacities and condensations consistent with a pattern of severe COVID-19 pneumonia; (B) CT scan disclosing bilateral adrenal gland hemorrhage; (C) left toes showing acral ischemic lesions; (D, E) angio-CT scan disclosing dorsalis pedis artery occlusion with 3D reconstruction.

He received hydroxychloroquine ( $400 \mathrm{mg}$ a day for 7 days) and azithromycin (500 $\mathrm{mg}$ on day 1 followed by $250 \mathrm{mg}$ for 4 more days) together with low flow oxygen for a couple of days. On day 12 , while fever and pulmonary involvement had improved, the patient suffered sudden abdominal pain revealing bilateral adrenal glands hemorrhage ( - Fig. 1B). INR was still correct (2.7), but we observed high levels of fibrinogen $(7 \mathrm{~g} / \mathrm{L})$ and D-dimers $>2 \mathrm{~N}(1,309 \mathrm{ng} / \mathrm{mL})$, and decreased antithrombin III activity (63\%). It was then decided to stop VKA and switch for low-molecular-weight heparin (enoxaparin $100 \mathrm{UI} / \mathrm{kg}$ twice a day). On day 17, painful acral ischemic lesions concerning left toes revealed dorsalis pedis artery occlusion (-Fig. 1C-E). No VTE was found in association, embolic causes were ruled out, and positivity for LA and APL-Abs was confirmed. Treatment was switched for intravenous continuous unfractionated heparin (anti-Xa target 0.5-0.7) and no other clinical or radiological thrombotic event occurred thereafter, excluding catastrophic APLS. The patient was eventually discharged on April 16 (day 27) under VKA and substitutive opotherapy for adrenal function.

In addition to an increased risk for adverse outcome in patients with prior cardiovascular disease, COVID-19 can also lead to an hypercoagulability state ${ }^{3}$ resulting in vascular and thrombotic events, such as ST-segment elevation coronary syndromes, ${ }^{4}$ pulmonary embolism, ${ }^{5}$ and disseminated intra- vascular coagulation. ${ }^{6}$ In this context, Tang et $\mathrm{al}^{7}$ reported the prognosis value of abnormal coagulation parameters (high $D$ dimer and fibrin degradation product levels). All these findings prompted the International Society on Thrombosis and Haemostasis to edit recommendations for anticoagulant therapy in COVID-19. ${ }^{8}$ The underlying cardiovascular risk factors, critical illness with hypoxemic conditions, hemostatic factors, and intense inflammatory response were considered initially to predispose to vascular events in COVID-19. Whether thrombosis is linked to some mechanisms specific to SARS-CoV-2 is now considered as a possibility but is still to be fully demonstrated. Interestingly, microthrombi have been found within the lungs of COVID-19 patients according to autopsy studies ${ }^{9}$ and also within skin lesions. ${ }^{10} \mathrm{~A}$ thrombotic microangiopathy might contribute to COVID-19 injury in some cases, in association with the activation of the alternative and lectin pathways of complement system ${ }^{11-13}$ and review. ${ }^{14}$ The complement system is even being considered as a target for therapy in COVID-19. ${ }^{15-17}$

In April, Zhang et $\mathrm{al}^{2}$ reported multiple cerebral infarctions in three COVID-19 patients with APL-Abs (aCL IgA, aß2GPI IgA and IgG). This presentation was suggestive of APLS but did not meet the criteria in terms of antibody isotype, titer, and persistence. ${ }^{18}$ Notably, APL-Abs (mostly aCL antibodies) may be detected in the course of viral infections and/or critical illness. In these settings, they 
may be the markers of endothelial damage and may inconstantly be associated with thrombotic events. ${ }^{19}$ Similarly, LA has been reported as a frequent feature of COVID-19, but ongoing heparin treatment may interfere with the LA detection and false-positive tests cannot be excluded. ${ }^{20}$ In this study, among 56 COVID- 19 patients, $45 \%$ were positive for LA and only $10 \%$ positive for aCL or a $32 \mathrm{GPI}$. Since then, other reports have discussed the pathogenic role of LA and/or APL detected in COVID-19 patients, considering that APL could be transient and were not always associated with thrombotic manifestations. ${ }^{21-23}$ However, some patients with higher and persistent levels of APL or multiple APL positivity may be more at risk of cerebral infarction during COVID-19. ${ }^{24}$

On the whole, in some patients, APL-Abs may be present prior to COVID-19, as a latent autoimmune condition that could be precipitated by SARS-CoV-2, as described in catastrophic APLS. ${ }^{1}$

Furthermore, in a model of in vitro engineered human blood vessel organoids, SARS-CoV-2 shows a special tropism for endothelium through the expression of its receptor angiotensin converting enzyme $2 .{ }^{25}$ In addition, lesions of endotheliitis appear to be induced by SARS-CoV-2, as demonstrated by the presence of viral particles within endothelial cells, together with inflammatory cells, resulting in apoptosis of these cells. ${ }^{26}$ Thus, as suggested in our observation, SARS-CoV-2 may favor the pathogenic effects of APL (including the activation of endothelial cells, monocytes, platelets, and complement), resulting in proinflammatory/prothrombotic states. In a broader view, the endothelial tropism of SARS-CoV-2 may also modify the clinical presentation of COVID-19 in susceptible patients and it might be hypothesized that it triggers flares of underlying vascular diseases.

In the present case, we can also speculate that SARS-CoV2 may have been responsible for thrombosis through a "twohit hypothesis" in a patient with persistent APL. Moreover, we cannot exclude that the modification of anticoagulant therapy (i.e., switch for low-molecular-weight heparin) may also have contributed to APLS flare. However, bilateral adrenal glands hemorrhage (a rare but typical feature of APLS) was observed prior to the switch, which suggests that the thrombotic process was already ongoing. So, taken together, our findings and literature data suggest that COVID-19 represents a high-risk condition for APLS flare.

If APL-Abs positivity can be an epiphenomenon under inflammatory and prothrombotic conditions, COVID-19 was herein responsible for disseminated thrombosis and a threat of catastrophic APLS, despite efficient anticoagulation.

\section{Authors' Contributions}

Each author contributed to the patient care. A.T.J.M. and P. G. wrote the first draft, and all the authors proofread it. The same was accepted in its last version.

\section{Conflict of Interest}

A.T.J.M. declares speaking fees from Sanofi, AstraZeneca, and Bristol Myers Squibb in the last 36 months. P.G. has been a medical expert for LFB (Laboratoire Français du Biofractionnement) and has received fees from Actelion,
Novartis, and Pfizer in the last 36 months. The other authors declare no conflict of interest.

\section{References}

1 Willis R, Pierangeli SS. Pathophysiology of the antiphospholipid antibody syndrome. Auto Immun Highlights 2011;2(02):35-52

2 Zhang Y, Xiao M, Zhang S, et al. Coagulopathy and antiphospholipid antibodies in patients with Covid-19. N Engl J Med 2020;382 (17):e38

3 Connors JM, Levy JH. Thromboinflammation and the hypercoagulability of COVID-19. J Thromb Haemost 2020;18(07):1559-1561

4 Bangalore S, Sharma A, Slotwiner A, et al. ST-segment elevation in patients with Covid-19 - a case series. N Engl J Med 2020;382(25): 2478-2480

5 Cui S, Chen S, Li X, Liu S, Wang F. Prevalence of venous thromboembolism in patients with severe novel coronavirus pneumonia. J Thromb Haemost 2020;18(06):1421-1424

6 Lillicrap D. Disseminated intravascular coagulation in patients with 2019-nCoV pneumonia. J Thromb Haemost 2020;18(04):786-787

7 Tang N, Li D, Wang X, Sun Z. Abnormal coagulation parameters are associated with poor prognosis in patients with novel coronavirus pneumonia. J Thromb Haemost 2020;18(04):844-847

8 Thachil J, Tang N, Gando S, et al. ISTH interim guidance on recognition and management of coagulopathy in COVID-19. J Thromb Haemost 2020;18(05):1023-1026

9 Barton LM, Duval EJ, Stroberg E, Ghosh S, Mukhopadhyay S. COVID-19 autopsies, Oklahoma, USA. Am J Clin Pathol 2020;153 (06):725-733

10 Gianotti R, Veraldi S, Recalcati S, Cusini M, Ghislanzoni M, Boggio F, Fox LP. Cutaneous clinico-pathological findings in three COVID19-positive patients observed in the metropolitan area of Milan, Italy. Acta Derm Venereol 2020;100(08):adv00124

11 Magro C, Mulvey JJ, Berlin D, et al. Complement associated microvascular injury and thrombosis in the pathogenesis of severe COVID-19 infection: a report of five cases. Transl Res 2020;220:1-13

12 Gao T, Hu M, Zhang X, et al. Highly pathogenic coronavirus N protein aggravates lung injury by MASP-2-mediated complement over-activation. Infectious Diseases (except HIV AIDS) 2020 (preprint). Available at: https://www.medrxiv.org/content/10.1101/ 2020.03.29.20041962v2.full.pdf. Accessed September 2, 2020

13 Cugno M, Meroni PL, Gualtierotti R, et al. Complement activation in patients with COVID-19: a novel therapeutic target. J Allergy Clin Immunol 2020;146(01):215-217

14 Chauhan AJ, Wiffen LJ, Brown TP. COVID-19: a collision of complement, coagulation and inflammatory pathways. J Thromb Haemost 2020

15 Risitano AM, Mastellos DC, Huber-Lang M, et al. Complement as a target in COVID-19? Nat Rev Immunol 2020;20(06):343-344

16 Diurno F, Numis FG, Porta G, et al. Eculizumab treatment in patients with COVID-19: preliminary results from real life ASL Napoli 2 Nord experience. Eur Rev Med Pharmacol Sci 2020;24 (07):4040-4047

17 Smith K, Pace A, Ortiz S, Kazani S, Rottinghaus S. A phase 3 openlabel, randomized, controlled study to evaluate the efficacy and safety of intravenously administered ravulizumab compared with best supportive care in patients with COVID-19 severe pneumonia, acute lung injury, or acute respiratory distress syndrome: a structured summary of a study protocol for a randomised controlled trial. Trials 2020;21(01):639

18 Miyakis S, Lockshin MD, Atsumi T, et al. International consensus statement on an update of the classification criteria for definite antiphospholipid syndrome (APS). J Thromb Haemost 2006;4 (02):295-306

19 Sène D, Piette J-C, Cacoub P. Antiphospholipid antibodies, antiphospholipid syndrome and infections. Autoimmun Rev 2008;7 (04):272-277 
e210 Flare of Antiphospholipid Syndrome in the Course of COVID-19 Maria et al.

20 Harzallah I, Debliquis A, Drénou B. Lupus anticoagulant is frequent in patients with Covid-19. J Thromb Haemost 2020;18(08): 2064-2065

21 Devreese KMJ, Linskens EA, Benoit D, Peperstraete H. Antiphospholipid antibodies in patients with COVID-19: a relevant observation? J Thromb Haemost 2020. doi: 10.1111/ jth.14994

22 Pineton de Chambrun M, Frere C, Miyara M, et al. High frequency of antiphospholipid antibodies in critically ill COVID-19 patients: a link with hypercoagulability? J Intern Med 2020. doi: 10.1111/ joim.13126
23 Siguret V, Voicu S, Neuwirth M, et al. Are antiphospholipid antibodies associated with thrombotic complications in critically ill COVID-19 patients? Thromb Res 2020;195:74-76

24 Xiao M, Zhang Y, Zhang S, et al. Brief report: anti-phospholipid antibodies in critically ill patients with coronavirus disease 2019 (COVID-19). Arthritis Rheumatol 2020. doi: 10.1002/art.41425

25 Monteil V, Kwon H, Prado P, et al. Inhibition of SARS-CoV-2 infections in engineered human tissues using clinical-grade soluble human ACE2. Cell 2020;181(04):905-913.e7

26 Varga Z, Flammer AJ, Steiger P, et al. Endothelial cell infection and endotheliitis in COVID-19. Lancet 2020;395(10234):1417-1418 